\title{
Prediction of Iron Ore Demand Based on Coupled Phase-Space Reconstruction and Neural Network
}

\author{
Xiaojun YAN, Zhiya CHEN \\ Business School of Central South University, Changsha, Hunan 410083, China
}

\begin{abstract}
The over capacity of steel and structure adjustment of iron production cause the producing elasticity. As the major raw material, the demand of iron ore fluctuates significantly and it brings great trouble for steel enterprise in business decision-making. In order to improve the management decisions, the steel enterprises must carry out the effective predictions of the iron ore demands. Based on the coupled phase space reconstruction and neural network, we proposed a prediction model of the iron ore demand, which first used the raw demand data for the coupled phase-space reconstruction, then trained these reconstructed data with the neural network, finally predicted the iron ore demand according to the predicted time. Besides, the iron ore quarter demand data at 2001-2011 from a typical steel enterprise was used for verifying this prediction model. Results show that this prediction model of the iron ore demand is easy to operate and its predicted data is reliable, which can provide theoretical guidance to the scientific and reasonable management decisions.
\end{abstract}

KEYWORD: chaos; coupled phase-space reconstruction; neural network; iron ore demand prediction

With the adjustment of construction, the over production of steel is seriously in China, and the steel enterprise has to manufacture flexibly according to the consumers' demand and market change, and it brings huge pressure for these enterprises in decision-making. On the one hand, enterprises should try to satisfy consumers' demand and enhance their satisfaction degree. On the other hand, enterprises should try to reduce the relative logistics costs because of the demand fluctuation. Therefore, how to effectively forecast consumers' demand, offer scientifically theoretical references for the management decision department, and plan as a whole in the whole enterprise and exert the maximum benefits of various resources in the enterprise will be the important content of the client relation management and the supply chain management.

There are many methods about the demand forecasting at present, such as the regression analysis [1], the Markov prediction [2], the Gray prediction, [3] and the artificial neural network (ANN) prediction [4-5]. The good adaptability, the nonlinearity, and the fault tolerance of ANN have been applied widely, but the train of the network needs sufficient sample space, and the specific forecasting function expression is hard to provide [6]. How to select the forecasting method which needs minimum data and has good fitting nonlinear function is the key problem of the demand forecasting. The phase-space reconstruction technology [7-10] in the chaos theory and the ANN are effective tools to deal with the problems about nonlinear system, and they also could be combined effectively. The phase-space reconstruction technology could display the concealed system state information in the time sequence, so the demand of the original data will be reduced, and the reconstructed data will be fitter for the sequent disposal by ANN.

\section{CHAOS THEORY}

The research of the chaos theory by the nonlinear dynamics is most systematic and rigorous up to now. In the nonlinear dynamics, though some definitions of theoretical judgment and standards of actual measurement only define the chaos from the mathematical and physical angle, but they are the base to establish and develop the chaos theory, where the Li-Yorke Theorem is the mathematical definition of chaos with large influences.

In 1975, Li Tianyan and Yorke JA first put forward the word of chaos, and they mathematically define the chaos in the article of "Period 3 means chaos", and it is called the definition of Li-Yorke now. 
Li-Yorke Theorem: Suppose $f(x)$ is the continuous self-mapping in $[a, b]$, and if has 3 period points, it has $\mathrm{n}$ period points for any positive integer $n$.

Definition of chaos: Suppose the continuous selfmapping $f: I \rightarrow I$ is contained in $R$, and $I$ is one subinterval in $R$, and if the uncountable set $S$ exists and it is contained in $I$.

(1) $S$ doesn't contain period points.

For any $X_{1}, X_{2} \in S\left(X_{1} \neq X_{2}\right)$,

$$
\begin{aligned}
& \limsup _{t \rightarrow \infty}\left|f^{t}\left(X_{1}\right)-f^{t}\left(X_{2}\right)\right|>0 \\
& \liminf _{t \rightarrow \infty}\left|f^{t}\left(X_{1}\right)-f^{t}\left(X_{2}\right)\right|=0
\end{aligned}
$$

Here, $\quad f^{t}(\bullet)=f(f(\cdots f(\bullet)))$ denotes $\quad t$-multiple function relation.

For any $X_{1} \in S$ and any period point of $f$, $P \in I$,

$$
\limsup _{t \rightarrow \infty}\left|f^{t}\left(X_{1}\right)-f^{t}(P)\right|>0
$$

So, $f$ is chaotic in $S$.

This definition is based on a set, but it shows the important characters of the chaos. First, countable infinite stable period solutions exist. Second, uncountable infinite stable non-periodic tracks exist. Finally, one unstable non-periodic track exists at least.

\section{MODELING APPROACHES OF THE NN FORECASTING MODEL OF THE PHASE- SPACE RECONSTRUCTION}

\subsection{Principle of phase-space reconstruction}

The phase-space reconstruction comes from the chaotic dynamics, and its basic principle is to display the system state information containing in the time sequence by the reconstruction technology. The chaotic time sequence signal disposal method is mainly based on the angle of the dynamics system, which is different with the traditional time sequence disposal method which is mainly to transmit and dispose the time sequence, and to acquire the information of the system in the time sequence, Pachard et al first put forward the phase-space reconstruction technology, and then Taken further developed it and proved it mathematically, and it is called as the phase-space reconstruction theorem (Takens Theorem).

For the dynamic system with $n$ dimensional variables (i.e. a set of $n$ first-order differential equations),

$$
\frac{\mathrm{d} x_{i}}{\mathrm{~d} t}=f_{i}\left(x_{1}, \ldots, x_{n}\right)(i=1,2, \ldots, n)
$$

By the method of elimination, it could be transformed as a n-order linear differential equation,

$$
x^{(n)}=f\left(x, x^{(1)}, \ldots x^{(n-1)}\right)
$$

After transformation, the track of the system is

$$
\vec{x}(t)=\left[x(t), x^{\prime}(t), \ldots, x^{(n-1)}(t)\right]
$$

It describes same dynamic problem, and it will evolve in the phase-space formed by the coordinate $x(t)$ with $(n-1)$-order $x^{\prime}(t), \ldots, x^{(n-1)}(t)$ and substitute this continuous variable $x(t)$ and its derivative without considering discontinuous time sequence and its $(n-1)$ time-lag displacement.

$$
\vec{x}(t)=\{x(t), x(t+\tau), \ldots \ldots, x(t+(n-1) \tau)\}
$$

Select the time lag $\tau$ as the large-scale research of the time sequence, which will ensure the coordinates to be linearly independent. Therefore, if (1) the dynamic system has single one state variable $x(t)$, and (2) its track evolves in the phase-space forming in the time displacement coordinate, so the attractor of the dynamic system could be reconstructed in the new phase-space with the lagged coordinates where its topological character will not be changed.

In the practice, for the appointed time sequence, $x\left(t_{0}\right), x\left(t_{1}\right), \ldots, x\left(t_{i}\right), \ldots x\left(t_{n}\right)$, it will generally be extended to three-dimensional or multiple-dimensional space, which could fully display all information contained in the time sequence, and that is the lag coordinate state space reconstruction method. The above time sequence will be extended as a phase distribution with $m$-dimensional phase space.

$$
\left[\begin{array}{cccccc}
x\left(t_{1}\right), & x\left(t_{2}\right), & \cdots, & x\left(t_{i}\right), & \cdots, & x\left(t_{n}\right), \\
x\left(t_{1}+\tau\right), & x\left(t_{2}+\tau\right), & \cdots, & x\left(t_{i}+\tau\right), & \cdots, & x\left(t_{n}+\tau\right), \\
x\left(t_{1}+2 \tau\right), & x\left(t_{2}+2 \tau\right), & \cdots, & x\left(t_{i}+2 \tau\right), & \cdots, & x\left(t_{n}+2 \tau\right), \\
\cdots, & \cdots, & \cdots, & \cdots, & \cdots, & \cdots, \\
x\left(t_{1}+(m-1) \tau\right) & x\left(t_{2}+(m-1) \tau\right), & \cdots, & x\left(t_{i}+(m-1) \tau\right), & \cdots, & x\left(t_{n}+(m-1) \tau\right),
\end{array}\right]
$$

Here, $\tau$ is the time lag, and each list in the above formula will form one phase point in the $m$ dimensional phase space, and any one phase point has $m$-dimensional components,

$$
\left[x\left(t_{i}\right), x\left(t_{i}+\tau\right), \cdots, x\left(t_{i}+(m-1) \tau\right)\right] \quad i=1,2, \cdots, n
$$

which denotes the state of certain instant in this system. And the link among phase points would describe the evolvement track of the system in the $m$-dimensional phase space.

\subsection{Parameter selection of phase-space reconstruction}

To reconstruct the phase space from the time sequence, the key technology is to correctly select the dimension amount of the embedded space $m$, and the time lag $\tau$. The selection of $\tau$ is not important principally, but because of the noise and estimation, the selection of proper $\tau$ is very important. If $\tau$ is too small and the correlation of 
each coordinate is too strong, tracks in the phase space will extrude to one place, and the information about attractor will not be displayed. If $\tau$ is too big, in the chaos and noise, the dynamics state at certain time and the dynamics state at next time will change drastically, so even simple geometric objects seem very complex. In the reconstructed phase space, it is very important to select the time lag $\tau$ and the embedded dimension $m$, and at the same time, this selection is difficult.

\subsubsection{Computing the delay time by the autocorrelation function method}

The main methods to solve the time lag include the autocorrelation function method and the mutual information method. The autocorrelation function method is selected in this article, and its advantages include that it could ensure the small correlation of various coordinate components in the phase space, and accord with the requirements to reconstruct the phase space, and the computation is simple. The concrete computation method could be described as follows.

For the time sequence of the variable $x(t)$, $x_{1}, x_{2}, \cdots, x_{n}$, the autocorrelation function expression is

$c(\tau)=\frac{n}{n-\tau} \frac{\sum_{i=1}^{n-\tau}\left(x_{i}-\mu\right)\left(x_{i+\tau}-\mu\right)}{\sum_{i=1}^{n}\left(x_{i}-\mu\right)^{2}}$

Where, $\tau$ is the time lag, and $\mu$ is the average of the time sequence.

The autocorrelation function denotes the times which subscripts are $i, i+\tau$, and the correlation or the similarity of the movement. The function image of the autocorrelation function about the time $\tau$ could be drew, and when the autocorrelation function descends to the first zero value, the value of $\tau$ could be the time lag embedded into the phase space. In addition, according to the numerical value experiment result, when the autocorrelation function descends to $\mathrm{t}_{1}-\frac{1}{e}$ of the initial value, the time $\tau$ could be the time lag of the reconstructed phase space.

\subsubsection{Computing the embedded dimensions by the Cao method}

This method is improved false zero method, for

$x_{i}=\left(x_{i}, x_{i+\tau}, \cdots, x_{i+(m-1) \tau}\right) \in R^{m}, i=1,2, \cdots$

Define: $a(i, m)=\frac{\left\|x^{(m+1)}(i)-x^{(m+1)}(j)\right\|}{\left\|x^{(m)}(i)-x^{(m)}(j)\right\|}$

Where, $x^{(m+1)}(i)$ is the $i$ 'th phase point in the $(m+1)$-dimensional reconstructed phase space, and $x^{(m+1)}(j)$ is the nearest neighboring point of $x^{(m+1)}(i)$, and $\|\cdot\|$ is the Euclidean distance. The average of all phase points $a(i, m)$ is

$E(m)=\frac{1}{n-m \tau} \sum_{i=1}^{n-m \tau} a(i, m)$

$E(m)$ only depends on the embedded dimension $m$ and the time lag interval $\tau$, and to study the change of the embedded dimension from $m$ to $m+1$ in the phase space, define

$F(m)=\frac{E(m+1)}{E(m)}$

If $F(m)$ goes to saturation with the increase of $m$, the value of $m$ is the minimum embedded dimension of the reconstructed phase space.

\subsection{Coupling NN forecasting}

$\mathrm{NN}$ is the adaptive nonlinear system which is associated by concurrent and highly correlative computation disposal unites, i.e. the neural cells, according to certain mode. Though the structure of each neural cell is very simple, but the neural cell system linked by numerous neural cells could implement abundant behaviors. NN has strong nonlinear mapping ability, and because of the concurrent disposal and strong nonlinear mapping ability of $\mathrm{NN}$, the $\mathrm{NN}$ could predict the behavior after it is trained by sufficient training samples. Therefore, the phase-space reconstruction technology could be used to confirm the input of the $\mathrm{NN}$ model, and the NN forecasting model based on the phase-space reconstruction could be established.

In the practice, according to the embedded dimension $m$ of the chaotic time sequence, $m-1$ could be selected as the node amount of the network input layer, and the node amount of the output layer is 1 . The input and the output of the networks respectively are

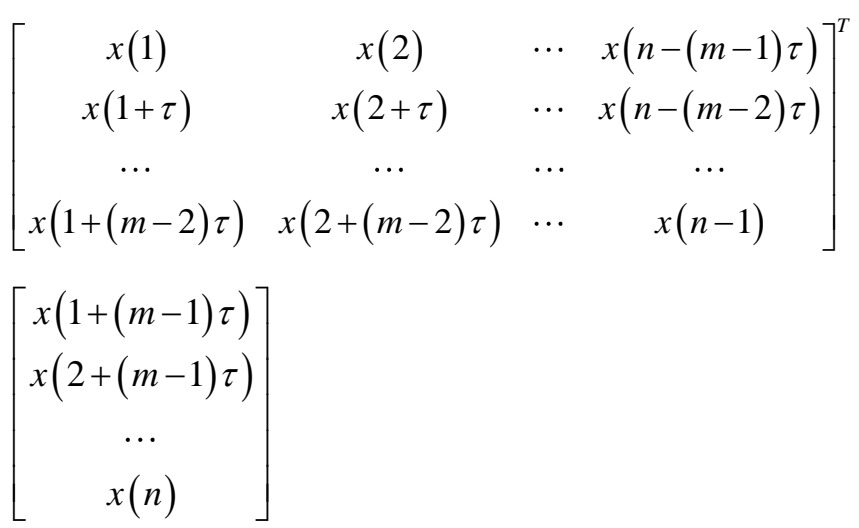

After confirming the input and output structure of the network, the study sample in the known time sequence could be abstracted to compose the training set to train the network until achieving certain error precision. 
To further forecast the behavior, the value of $x(n+1)$ needs to be known. Input $[x(n+1-(m-1) \tau), x(n+1-(m-2) \tau), \cdots, x(n+1-\tau)]$ into the trained $\mathrm{NN}$ model, the value of $x(n+1)$ could be obtained. In the same way, by changing the input data, the values of $x(n+2), x(n+3), \cdots$ could be obtained.

\section{THE APPLICATION OF THE NN}

FORECASTING MODEL BASED ON THE PHASE-SPACE RECONSTRUCTION IN THE PRACTICE

The forecasting example is the iron ore demands of one steel enterprise from 2006 to 2014, and as seen in Table 1, all data come from the actual survey.

Table 1 Bauxite demand volume from 2006 to 2011

\begin{tabular}{|c|c|c|c|c|}
\hline \multirow{2}{*}{ Year } & \multicolumn{4}{|c|}{ Demand (10 thousand T) } \\
\cline { 2 - 5 } & $1^{\text {st }}$ quarter & $2^{\text {nd }}$ quarter & $3^{\text {rd }}$ quarter & $4^{\text {th }}$ quarter \\
\hline 2006 & 108 & 129 & 113 & 120 \\
\hline 2007 & 127 & 135 & 137 & 142 \\
\hline 2008 & 146 & 150 & 156 & 153 \\
\hline 2009 & 157 & 175 & 179 & 171 \\
\hline 2010 & 171 & 185 & 179 & 183 \\
\hline 2011 & 183 & 192 & 185 & 202 \\
\hline 2012 & 204 & 212 & 203 & 198 \\
\hline 2013 & 203 & 209 & 212 & 152 \\
\hline 2014 & 122 & 159 & 183 & 227 \\
\hline
\end{tabular}

\subsection{Reconstructing the phase-space}

Aiming at the data of demand, the autocorrelation function method and the Cao method are used to compute the time lag and the embedded dimension by the Matlab programming. The time lag computation result of the demand is seen in Figure 1, and the computation result of the embedded dimension is seen in Figure 2. From Figure 1, for the point which first arrives at the zero, because $c(11)=0.0644$ and $c(12)=-0.0221$, if the corresponding time lag $\tau$ of the autocorrelation function value which first arrives at zero should be 12 , but this value is too big, and after forecasting the time lag of this value in the reconstructed phase space, the value is also too begin the result. Therefore, the descending tendency of the autocorrelation function is adopted to select the time lag $\tau$ in the reconstructed phase space. After computing, $\quad c(2)=0.5863$. After $c(2)$, the autocorrelation function descends slowly with the increase of $\tau$, so in this article, $\tau=2$. From Figure2, when the embedded dimension $m=4$, the value of $F(m)$ goes to saturation, so $m=4$.

\subsection{Phase-space reconstruction and NN coupling forecasting}

According to the embedded dimension $m$ (4) and the time lag $\tau$ (2), reconstruct the time sequence. Take $m-1$ as the input node amount of the network, and take the output node amount as 1, and construct one NN model with one input layer, two concealed layers, and one output layer (seen in Figure 3). Use reconstructed data to train the $\mathrm{NN}$, and the checking error precision is 0.001 . Figure 4 is the error change process of the network training and the checking process.

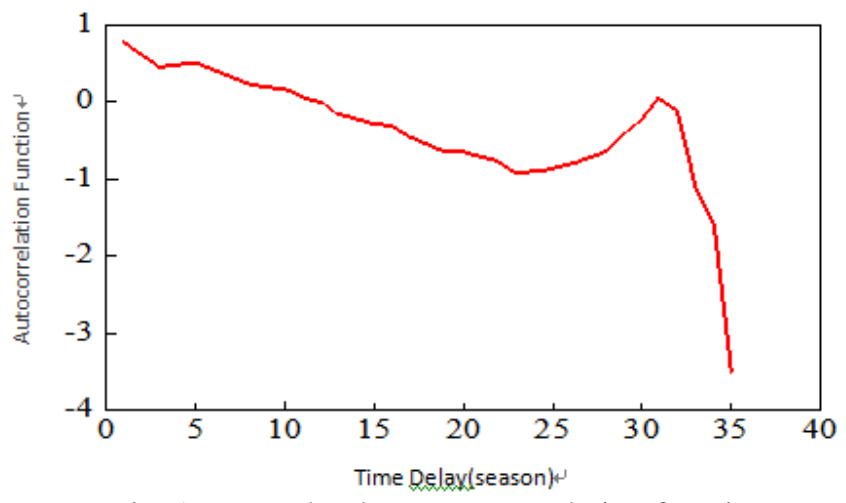

Fig. 1 Demand volume autocorrelation function

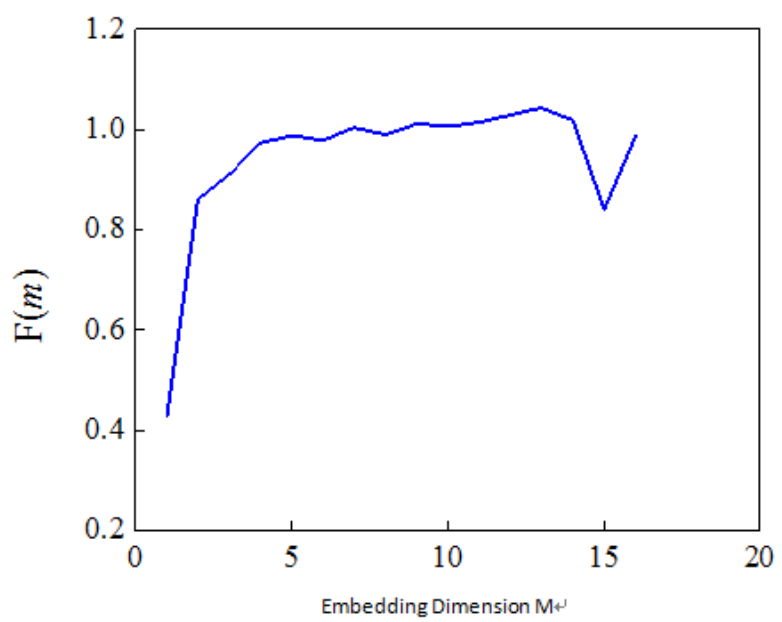

Fig. 2 Demand volume correlation dimension

When the network is trained successfully, the coupling forecasting model of the phase-space reconstruction and $\mathrm{NN}$ is established. Based on this model, forecast the demand, and compute the forecasting values of the demands from 2011 to 2014, and further compute the residuals and relative errors (seen in Table 2).

\subsection{Analysis of forecasting result}

The average relative error of the forecasting demand is $1.21 \%$, and good effect could be achieved. At the same time, the mutation factor could not be display well in the $\mathrm{NN}$ forecasting model based on the phase-space reconstruction. For example, the demand descends drastically in the fourth quarter of 2013, and the big gap between the forecasting data 
with the actual data in the fourth quarter of 2013 obviously exist, and the relative error is 6.68 , which far exceeds the average relative error $1.21 \%$.

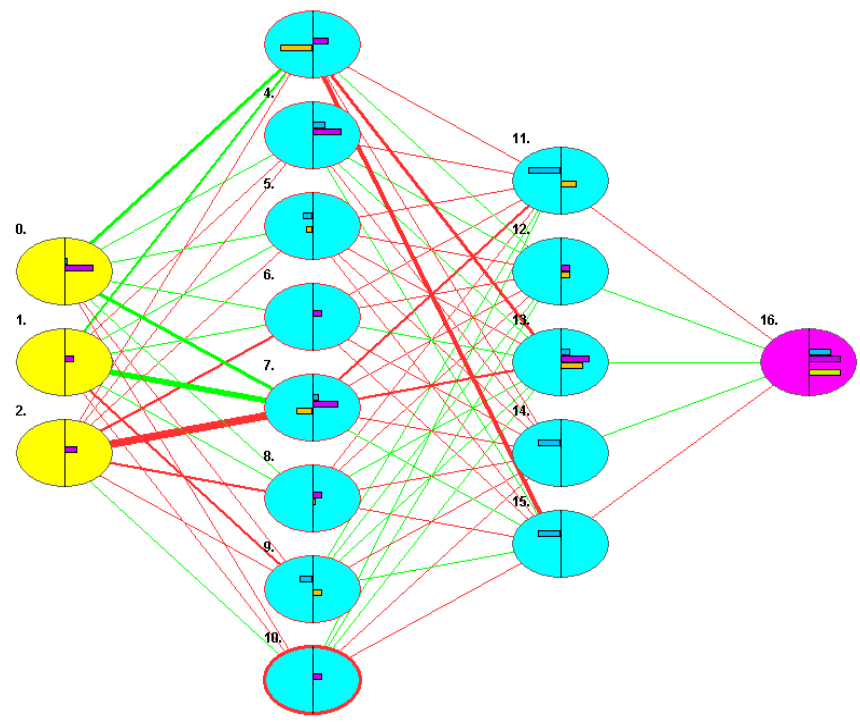

Fig. 3 Neural network structural diagram

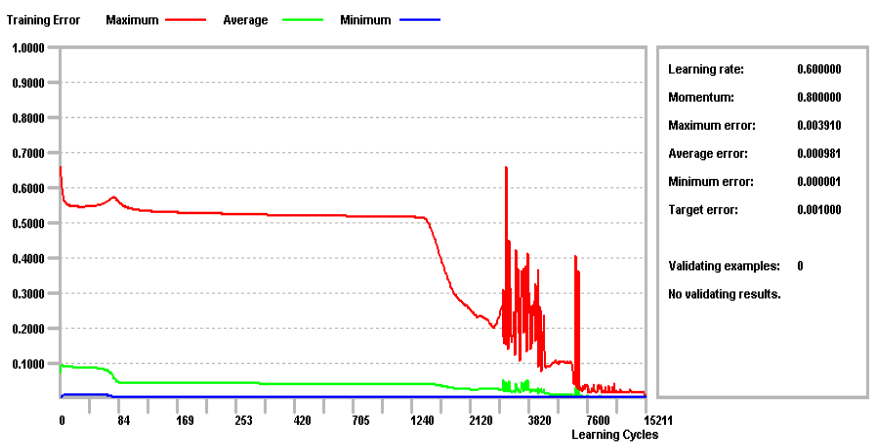

Fig. 4 Network study validation error convergence process curve

Table 2 Calculating value of residual and relative error s of original volume and forecasting volume

\begin{tabular}{|c|c|c|c|c|c|}
\hline Year & Quarter & $\begin{array}{l}\text { Original } \\
\text { volume } \\
/(10 \\
\text { thousand } \\
\text { Ton })\end{array}$ & $\begin{array}{c}\text { Forecasting } \\
\text { volume } \\
/(10 \\
\text { thousand } \\
\text { Ton) } \\
\end{array}$ & $\begin{array}{l}\text { Residual } \\
\text { Error } \\
/(10 \\
\text { thousand } \\
\text { Ton) } \\
\end{array}$ & $\begin{array}{c}\text { Relative } \\
\text { Error } \\
/(\%)\end{array}$ \\
\hline \multirow{4}{*}{2011} & $1^{\text {st }}$ & 183 & 180.18 & 2.82 & 1.54 \\
\hline & $2^{\text {nd }}$ & 192 & 188.83 & 3.17 & 1.65 \\
\hline & $3^{\text {rd }}$ & 185 & 183.63 & 1.37 & 0.74 \\
\hline & $4^{\text {th }}$ & 202 & 204.10 & -2.10 & 1.04 \\
\hline \multirow{4}{*}{2012} & $1^{\text {st }}$ & 204 & 198.93 & 5.07 & 2.49 \\
\hline & $2^{\text {nd }}$ & 212 & 205.91 & 6.09 & 2.87 \\
\hline & $3^{\mathrm{rd}}$ & 203 & 200.64 & 2.36 & 1.16 \\
\hline & $4^{\text {th }}$ & 198 & 194.53 & 3.47 & 1.75 \\
\hline \multirow{4}{*}{2013} & $1^{\text {st }}$ & 203 & 198.98 & 4.02 & 1.98 \\
\hline & $2^{\text {nd }}$ & 209 & 206.11 & 2.89 & 1.38 \\
\hline & $3^{\mathrm{rd}}$ & 212 & 208.66 & 3.34 & 1.58 \\
\hline & $4^{\text {th }}$ & 152 & 141.84 & 10.16 & 6.68 \\
\hline \multirow{4}{*}{2014} & $1^{\text {st }}$ & 122 & 126.09 & -4.09 & 3.35 \\
\hline & $2^{\text {nd }}$ & 159 & 162.44 & -3.44 & 2.16 \\
\hline & $3^{\text {rd }}$ & 183 & 179.28 & 3.72 & 2.03 \\
\hline & $4^{\text {th }}$ & 227 & 226.88 & 0.12 & 0.05 \\
\hline
\end{tabular}

\section{CONCLUSIONS}

The demand of iron ore is influenced by multiple layers and factors, to exactly forecast the demand, large information needs to be collected, and various influencing factors should be considered. In this article, the coupling ANN model based on the phasespace reconstruction technology in the chaos theory is established, and the forecasting result shows that this model has certain advantages to forecast the demand of alumina. The chaos is a kind of phenomenon and behavior, and $\mathrm{NN}$ is a special nolinear network structure to approach the behavior, and both have their own advantages, and the phasespace reconstruction technology in the chaos theory is introduced in $\mathrm{NN}$ to establish the chaotic NN model which could strengthen the ability of NN to study and approach the nonlinear mapping.

\section{REFERENCES}

[1] Shizhu Li, Dongmei Li, Wuxiang Tang. Quantitative analysis for single index efficiency of Chinese regional science output. Science of science and management of S\&T, 2003. 3: 14-18.

[2] Lanfang Liu. The application of forecasting of Markov chain in enterprise management. Science and Management, 1995, 15(2): 58-59.

[3] Kai Peng, the transaction of railway container based on grey forecasting method. Railway Procurement and Logistics, 2009, 6: 47-48.

[4] Qihong Zhu, Gang Zhang, Enterprise Knowledge Management Model based on artificial neural network. Science of science and management of S\&T 2003, 8: 3234.

[5] Xue yang, Jianhua Li, Baoying Cheng, Scientific demand forecasting and research based on artificial neural network. Science of science and management of S\&T, 2004, 12: 29-32.

[6] Zhang, Li-ming. (1993). Artificial Neural Networks Models and Its Applications. Shanghai: Fudan University Press.

[7] Li T Y, Yorke J. Period 3 implies chaos. Amer. Math. Monthly, 1975, 82: 985-992.

[8] Lv J H, Lu J A, Chen S H. Chaotic Time Series Analysis and Its Application. Wuhan: Wuhan University Press, 2002.

[9] Packard N H, Crutchfield J P, Farmer J D, et al. Geometry From a Time Series. Physical Review Letters, 1980, 45(9):712-716.

[10] Takens F. Detecting Strange Attractors in Turbulence, Lecture Notes in Mathematics. Berlin: Springer-Verlag, 1981, 898: 366-381.

\section{ABOUT AUTHOR}

Yan Xiaojun(1970 ), male, born in Jiaozuo, Henan. Dr. of Central South University (Changsha, Hunan, 410083) majors in Logistics Management.

E-mail: xj_yan@chalco.com.cn 